\title{
Visual dominance and attention: The Colavita effect revisited
}

\author{
SCOTT SinNeTt \\ Universitat de Barcelona, Barcelona, Spain \\ Charles Spence \\ University of Oxford, Oxford, England \\ AND \\ SALVAdOR SOTO-FARACo \\ ICREA and Universitat de Barcelona, Barcelona, Spain
}

\begin{abstract}
Under many conditions, humans display a robust tendency to rely more on visual information than on other forms of sensory information. Colavita (1974) illustrated this visual dominance effect by showing that naive observers typically fail to respond to clearly suprathreshold tones if these are presented simultaneously with a visual target flash. In the present study, we demonstrate that visual dominance influences performance under more complex stimulation conditions and address the role played by attention in mediating this effect. In Experiment 1, we show the Colavita effect in the simple speeded detection of line drawings and naturalistic sounds, whereas in Experiment 2 we demonstrate visual dominance when the task targets (auditory, visual, or bimodal combinations) are embedded among continuous streams of irrelevant distractors. In Experiments 3-5, we address the consequences of varying the probability of occurrence of targets in each sensory modality. In Experiment 6 , we further investigate the role played by attention on visual dominance by manipulating perceptual load in either the visual or the auditory modality. Our results demonstrate that selective attention to a particular sensory modality can modulate - although not completely reverse - visual dominance as illustrated by the Colavita effect.
\end{abstract}

When confronted with stimuli coming from different sensory modalities, humans often rely on the modality that is most precise or accurate for the given task (see, e.g., Ernst \& Bülthoff, 2004; Welch \& Warren, 1980; but see also Battaglia, Jacobs, \& Aslin, 2003). Both everyday experience and the available empirical evidence support the widely held impression that vision is typically the dominant sensory modality for humans in many situations (Posner, Nissen, \& Klein, 1976; Rock \& Harris, 1967; Rock \& Victor, 1964; see also Cooper, 1998; Hohnsbein, Falkenstein, Hoormann, \& Blanke, 1991; Klein, 1977; Quinlan, 2000), although it is possible, under certain conditions, to demonstrate sensory dominance by the auditory and/or somatosensory systems (Ernst \& Banks, 2002; Ernst, Banks, \& Bülthoff, 2000; Lederman \& Abbott, 1981; Morein-Zamir, Soto-Faraco, \& Kingstone, 2003; Sekuler, Sekuler, \& Lau, 1997; Shams, Kamitani, \& Shimojo, 2000). Posner et al. (1976) argued that visual dominance might represent a by-product of attentional processes, hypothesizing that humans have a strong tendency to actively (i.e., endogenously) attend to visual events as a means of compensating for the poor alerting properties of the visual system (in comparison with the auditory or tactile system; see also Klein, 1977; Spence, Nicholls, \& Driver, 2001; Spence, Shore, \& Klein, 2001).

The dominance of the visual modality is not confined to humans. Shapiro, Jacobs, and LoLordo (1980) suggested that many other species may also be visually dominant under normal (i.e., nonaroused) conditions, since a majority of biologically important information is received visually. Indeed, visual dominance effects over audition have now been reported in cows (Uetake \& Kudo, 1994), pigeons (Foree \& LoLordo, 1973; Kraemer \& Roberts, 1985; Randich, Klein, \& LoLordo, 1978), and rats (Meltzer \& Masaki, 1973) with the use of food acquisition procedures (operant conditioning; see also Partan \& Marler, 1999). Interestingly, both animal and human subjects appear to "switch" their attention more toward the auditory modality under conditions of high arousal in order to react more rapidly to potential threats. For example, audition appears to be dominant for controlling avoidance behaviors, such as preventing electric shocks (see, e.g., Foree \& LoLordo, 1973; Gilbert, 1969; Shapiro et al., 1980). Thus, in the case of animal research, vision seems to be dominant for certain behaviors, such as appetitive behaviors, whereas audition appears to be more dominant for

S. Sinnett, ss@psych.ubc.ca 
others, such as avoidance behaviors. The fact that visual dominance in animals can be reduced under the appropriate behavioral conditions suggests a role of attention in explaining why vision appears to be the dominant sense under normal conditions, supporting Posner et al.'s (1976) original proposition.

Colavita (1974) described one of the most dramatic examples of visual dominance, or prepotency (see Posner, Nissen, \& Ogden, 1978; Welch \& Warren, 1986, for early reviews of the phenomenon of visual dominance). In Colavita's study, participants were asked to press one button whenever they heard a tone, and another button whenever they saw a light. In the majority of trials, only one stimulus (a tone or a light) was presented unpredictably, and, as one would expect, participants responded both rapidly and accurately. A few trials (5 out of 35) interspersed throughout the experiment were bimodal, consisting of the simultaneous presentation of the tone and the light. Strikingly, in these bimodal trials, participants almost always failed to respond to the sound, pressing only the visual response button on 49 of 50 trials, across all 10 participants tested in the experiment. Furthermore, a number of the participants reported that they did not even hear the auditory stimulus on the bimodal test trials on which they had responded to the light (16 of 49 trials). This tendency to respond only to the visual event was particularly surprising given that response latencies for unimodal auditory and visual stimuli were equivalent (297 vs. $299 \mathrm{msec}$, respectively). Moreover, when presented in separate blocks of experimental trials (i.e., under conditions of focused attention), participants typically responded more rapidly to the auditory stimulus than to the visual stimulus (Experiment 1, 179 vs. $197 \mathrm{msec}$, respectively).

In his pioneering study, Colavita (1974) informed participants that the rare bimodal trials occurring during the course of the experimental session were "accidental" and gave no specific instruction about how participants should respond on such trials - even going so far as to apologize to the participants for their very occurrence! Although this was done in an attempt to keep participants from realizing the true purpose of the experiment, it does raise the possibility that task demands and/or experimenter expectancy effects may have contributed to the pattern of results obtained (i.e., Intons-Peterson, 1983; Orne, 1962; Pierce, 1908). ${ }^{1}$ Moreover, in subsequent follow-up experiments (see, e.g., Egeth \& Sager, 1977), the auditory and visual stimuli were presented from different spatial locations. Consequently, spatial attention may have played a role in determining the results, given the possible preference of observers for responding to stimuli presented at or near fixation (usually the visual event), rather than to stimuli presented elsewhere, such as inside the head (when presented over headphones) or from peripheral loudspeakers. ${ }^{2}$

The present study had two goals. The first was to extend the Colavita (1974) visual dominance effect to more complex situations. The majority of studies that have investigated the Colavita effect have used comparatively simple events - such as brief auditory beeps and light flashes - and have required that subjects passively wait for the occurrence of a stimulus requiring imperative response (see Egeth \& Sager, 1977; Hohnsbein et al., 1991; Quinlan, 2000; Shapiro, Egerman, \& Klein, 1984; Shapiro \& Johnson, 1987). We extended the investigation of visual dominance to situations involving the presentation of more complex stimuli in a task involving the search for predetermined targets (pictures and sounds) embedded amongst a stream of distractor stimuli. Our reasoning was that this procedure should increase the overall perceptual load of the task (Lavie, 2005) in comparison with the simple detection of beeps and flashes used previously (see Colavita, 1974), perhaps representing everyday life multisensory contexts more accurately (see also Basil, 1994).

This brings us to the second goal of the present study, which was to address the role that attention may play in visual dominance. Posner et al. (1976) tried to explain visual dominance as a by-product of the attentional system's compensating for the poor alerting abilities inherent in the visual system. However, in a series of experiments, Colavita and colleagues (Colavita, 1974; Colavita, Tomko, \& Weisberg, 1976; Colavita \& Weisberg, 1979) claimed that the phenomenon actually has a sensory basis instead, occurring regardless of the allocation of a participant's attention.

As an improvement over Colavita's (1974) original experimental design, participants in the present study were provided with three response keys corresponding to auditory, visual, and bimodal targets. The participants in all previous studies of the visual dominance effect by Colavita (1974; Colavita et al., 1976; Colavita \& Weisberg, 1979) were provided with just two response keys (auditory and visual), thereby leaving open the possibility of motor interference on bimodal trials, in which participants were instructed to press both response keys at the same time. By providing a third key for bimodal responses, any possible role of motor conflict at the response selection stage was reduced. Finally, we also eliminated the potential confounds introduced by the spatial layout of the stimuli in previous studies by presenting our auditory and visual events from the same spatial location.

Somewhat confusingly, the exact measure of visual dominance in the paradigm introduced by Colavita has varied across subsequent studies. In Colavita's (1974, p. 411; Colavita et al., 1976, p. 25) original studies, the visual dominance effect was clearly defined as the failure of participants to respond to a sound when it was paired with a visual stimulus that would otherwise elicit a response when presented in isolation. Although Colavita (1974, p. 411) also reported a trend across all experiments for auditory responses to be faster than visual responses (185 vs. 197 msec, respectively) when presented in separate (unimodal) experiment blocks, the error data can be interpreted clearly as long as the visual response latencies are not significantly faster than the auditory response latencies.

Egeth and Sager (1977) introduced a second definition of visual dominance. In their study (Experiment 2), response latencies were compared between auditory and visual erroneous responses to bimodal targets, showing that, as RTs to auditory errors on bimodal trials were lon- 
ger, the presence of the visual stimulus interfered in the perceptual processing of the auditory stimulus. Egeth and Sager (1977) also presented additional data in line with Colavita's (1974): When presented in separate blocksthat is, in conditions of focused attention - response latencies were shorter for auditory targets in comparison with visual targets; whereas, when mixing auditory and visual targets in the same block, response latencies did not differ statistically. In all of the experiments reported here, unimodal auditory and visual targets were interspersed within the same block. Therefore, the extent of visual dominance will be gauged in terms of the percentage of visually based errors relative to auditory-based errors on bimodal trials.

In Experiment 1, we tested the basic Colavita visual dominance effect using stimuli that were more complex than the beeps and flashes used in previous studies. We controlled for the spatial location of the stimuli presented in the two sensory modalities, as opposed to using headphones (Egeth \& Sager, 1977), informed the participants clearly about the possible occurrence of bimodal trials, and provided a three-alternative response set that included one response key for each of the unimodal targets plus another response key for their combination (for bimodal targets). If the Colavita visual dominance effect extends to the processing of complex stimuli, we would expect to see an imbalance in errors for bimodal targets, with the visual response being given significantly more often than the auditory response.

\section{EXPERIMENT 1}

\section{Method}

Participants. Twenty-four students from the University of Barcelona participated in Experiment 1 in exchange for course credit. All reported normal hearing and normal or corrected-to-normal vision and were naive about the purpose of the experiment.

Apparatus and Materials. Ten line drawings of common objects chosen from the Snodgrass and Vanderwart (1980) database were used in the visual stream. The pictures were rotated $30^{\circ}$ to the left or right to ensure task difficulty (see Rees, Russell, Frith, \& Driver, 1999). A set of 10 familiar sounds was selected from a database of 103 sounds (downloaded from www.al freesoundeffects .com, 01/02/2003) on the basis of their clarity and familiarity, as rated by three reviewers. All of the sounds were edited to a duration of $350 \mathrm{msec}$ and equivalent average amplitude using Cool Edit software (Syntrillium Software Corp.). The pictures and sounds were presented and randomized using EXPE6 software (Pallier, Dupoux, \& Jeannin, 1997). Loudspeakers placed directly beside the computer monitor were used to present the sounds at an average level of $60 \mathrm{~dB}$, as measured from the participant's ear position. This stimulus configuration ensured that the auditory and visual stimuli appeared to originate from the same spatial location.

Procedure. The participants sat approximately $60 \mathrm{~cm}$ from a computer screen in a dimly lit, sound-attenuated room. They were instructed to monitor the auditory and visual streams and to respond as rapidly as possible to the unimodally presented auditory and visual targets or to the bimodal combination of both targets. The participants had to make one of three keypress responses on the keyboard as rapidly as possible each time they saw the visual target, heard the auditory target, or perceived both targets presented at the same time. The three different response keys ("J," "K," "L") were counterbalanced across participants for each response type: visual, auditory, or bimodal. The streams were presented continuously until all targets had been presented. Targets occurred on average once every $7.5 \mathrm{sec}$ (ranging from 5 to $10 \mathrm{sec}$ ) and were separated by a combination of silence and a blank screen. Visual and auditory targets were equiprobable (44 of each type), whereas bimodal targets were presented 22 times (i.e., $40 \%$ auditory targets, $40 \%$ visual targets, and $20 \%$ bimodal targets), thereby increasing the proportion of bimodal trials by more than a factor of four in comparison with Colavita's (1974) original study. The participants were given a training session containing five targets - two visual, two auditory, and one bimodal - before the main experimental session. The training session was repeated until the participant felt comfortable with the task.

\section{Results and Discussion}

The RT data for correct responses to bimodal and unimodal targets, as well as the error data from the bimodal trials, were analyzed. Specifically, errors on bimodal targets consisted of one of three types of mistakes: a unimodal visual response, a unimodal auditory response (i.e., responses with the unimodal keys rather than with the required bimodal response key), or a miss. The participants never made an inappropriate response on either type of unimodal target trial. Misses were infrequent (4\% for unimodal auditory trials, $4 \%$ for unimodal visual trials, and $2 \%$ for bimodal trials). The error rate for bimodal target trials was $13 \%$ overall and, critically, participants made erroneous unimodal visual responses significantly more often than erroneous unimodal auditory responses $[7.8 \%$ vs. $2.7 \% ; t(23)=2.3, p=.032$; see Figure $1 \mathrm{~A}]$. In terms of their response latencies, participants responded significantly more rapidly to unimodal auditory or visual targets than to bimodal targets $[t(23)=2.6, p=.018$; and $t(23)=2.6, p<.016$, respectively]. Finally, RTs to auditory targets $(785 \mathrm{msec})$ were not significantly different from RTs to visual targets $(775 \mathrm{msec})[t(23)=.5, p=$ .64; see Figure 1B].

The main result to emerge from the analysis of Experiment 1 was that after bimodal stimulation, participants exhibited a tendency to erroneously press the visual response key more often than the auditory response key (a $5.1 \%$ difference). These results demonstrate that the Colavita effect (one example of the phenomenon of visual dominance) extends beyond the perception of simple stimuli, such as the brief tones and light flashes used in a number of previous studies (see, e.g., Colavita, 1974; Colavita et al., 1976; Colavita \& Weisberg, 1979; Egeth \& Sager, 1977; Quinlan, 2000), to affect the processing of more complex stimuli. In addition, the trend in the response latency data seen throughout Colavita's original experimentation - namely, that RTs to bimodal targets are slower than those to either auditory or visual unimodal targets, with no differences in unimodal RTs, when presented within the same block (Colavita, 1974; Colavita et al., 1976; Colavita \& Weisberg, 1979; Egeth \& Sager, 1977) — was also observed here.

Experiment 1 produced a relatively small number of errors on the bimodal trials (13\% overall) in comparison with those reported by Colavita and his colleagues in their original studies (Colavita, 1974; Colavita et al., 1976; Colavita \& Weisberg, 1979). The methodological improvements incorporated into the present study, such as (1) informing participants of the possibility of bimodal 

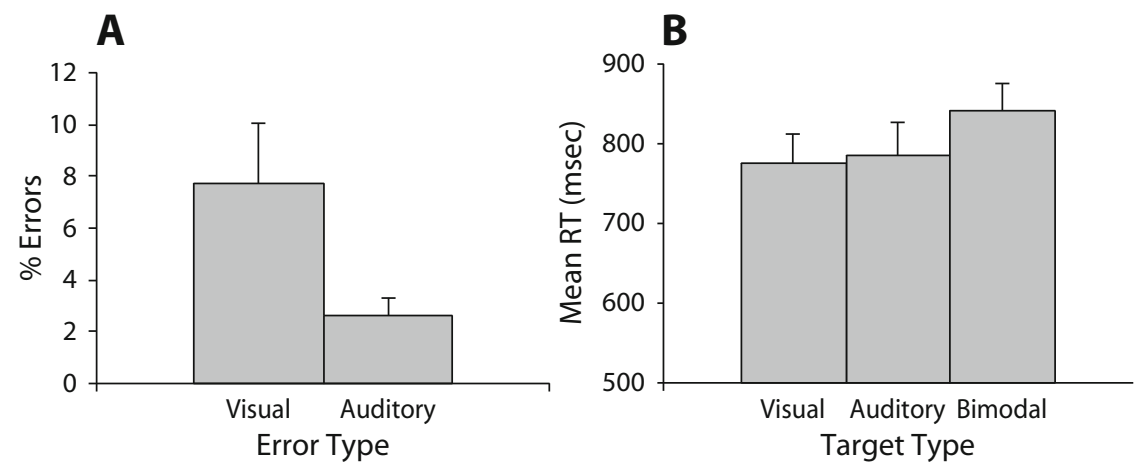

Figure 1. Results of Experiment 1. (A) The distribution of errors displayed as percentages of unimodal visual or unimodal auditory responses to bimodal trials. (B) Average RTs across the 3 target types (visual, auditory, and bimodal). The error bars represent the standard error of the mean for each condition.

targets, (2) including a greater proportion of bimodal trials, and (3) using a third, unique response key for these trials, might account for this reduction in the magnitude of the effect. In fact, our experiment provides the first demonstration of the Colavita effect incorporating a specific key for bimodal target stimuli to minimize the possible role of motor conflict. That is, participants in the present study were able to choose the bimodal response using a specified response key, rather than being forced to choose one of the two unimodal response keys with no instructions about how to respond to bimodal trials, or else having to respond by pressing both the auditory and visual target response keys simultaneously (Colavita, 1974). We believe that our experimental manipulation may therefore better control for the possibility of experimenter expectancy effects or task demands artificially contributing to the observed pattern of results (see, e.g., Intons-Peterson, 1983; Orne, 1962; Pierce, 1908).

After successfully replicating the Colavita effect in Experiment 1 , using stimuli that were more complex than those that had been used previously, we attempted to extend these results to a search situation with serially presented stimulus arrays in Experiment 2. This procedure was designed to increase the overall perceptual load of the participant's task (see Lavie, 2005), perhaps creating a testing situation that better represents everyday experience with multisensory stimuli (see Basil, 1994). If the Colavita visual dominance effect can be demonstrated to affect responses to complex stimuli in a task requiring participants to search for targets, we would once again expect to see an imbalance in errors for bimodal targets, with the visual response being given more frequently.

\section{EXPERIMENT 2}

\section{Method}

Participants. Fifty-four students from the University of Barcelona participated in Experiment 2 in exchange for course credit. All reported normal hearing and normal or corrected-to-normal vision, and were naive about the purpose of the experiment.

Apparatus and Materials. Forty additional line drawings of common objects (Snodgrass \& Vanderwart, 1980) were added to the original 10 drawings from Experiment 1 to create a total of 50 line drawings. As in Experiment 1, the pictures were rotated $30^{\circ}$ to the left or right to ensure task difficulty. The original set of 10 familiar sounds used in Experiment 1 was expanded to a set of 50; the additional 40 sounds were also selected from the same database of 103 sounds. As in Experiment 1, sounds were selected on the basis of their clarity and familiarity (using the same three reviewers). All other stimulus parameters remained the same as for Experiment 1.

Procedure. Rather than simply waiting for the appearance of any unimodal or bimodal target and responding accordingly, participants in this experiment were instructed to actively monitor the auditory and visual streams and to respond to predefined auditory and visual targets (specifically, the sound of a cat meowing or the picture of a stoplight), or to the combination of both targets. The pictures and sounds were presented synchronously for $350 \mathrm{msec}$ followed by an empty interstimulus interval (ISI) of $150 \mathrm{msec}$, the SOA being $500 \mathrm{msec}$ (see Figure 2). The participants had to make one of three keypress responses on the keyboard as quickly as possible each time they saw the visual target (stoplight), heard the auditory target (cat meow), or perceived both targets presented at the same time. The three different response keys ("J," "K," "L") were counterbalanced across participants for each response type: visual, auditory, or bimodal. As in Experiment 1, targets occurred on average every $7.5 \mathrm{sec}$, but were now separated by 10 to 20 items, instead of by silence and a blank screen. The proportion of visual, auditory, and bimodal targets was the same as in Experiment 1: $40 \%$ auditory targets, $40 \%$ visual targets, and $20 \%$ bimodal targets. The participants were given a training session containing five targets (two visual, two auditory, and one bimodal) before the main experimental session. Again, the training session was repeated until the participant felt comfortable with the task.

\section{Results and Discussion}

The RT data, as well as the error data from the bimodal target trials, were analyzed in the same way as in Experiment 1 . False alarm rates for nontarget trials were less than $0.01 \%$. Misses were slightly more frequent than in Experiment 1 , perhaps because of the increased perceptual load of the task (11\% for unimodal auditory target trials, $10 \%$ for unimodal visual target trials, and $0.8 \%$ for bimodal target trials). Errors were frequent on the bimodal target trials (49.4\% error rate overall) and, critically, participants made erroneous unimodal visual responses significantly more often than erroneous unimodal auditory responses $[27.8 \%$ vs. $20.5 \%, t(53)=2.1, p=.038$; see Figure $3 \mathrm{~A}]$. In terms of the RT data, participants responded signifi- 


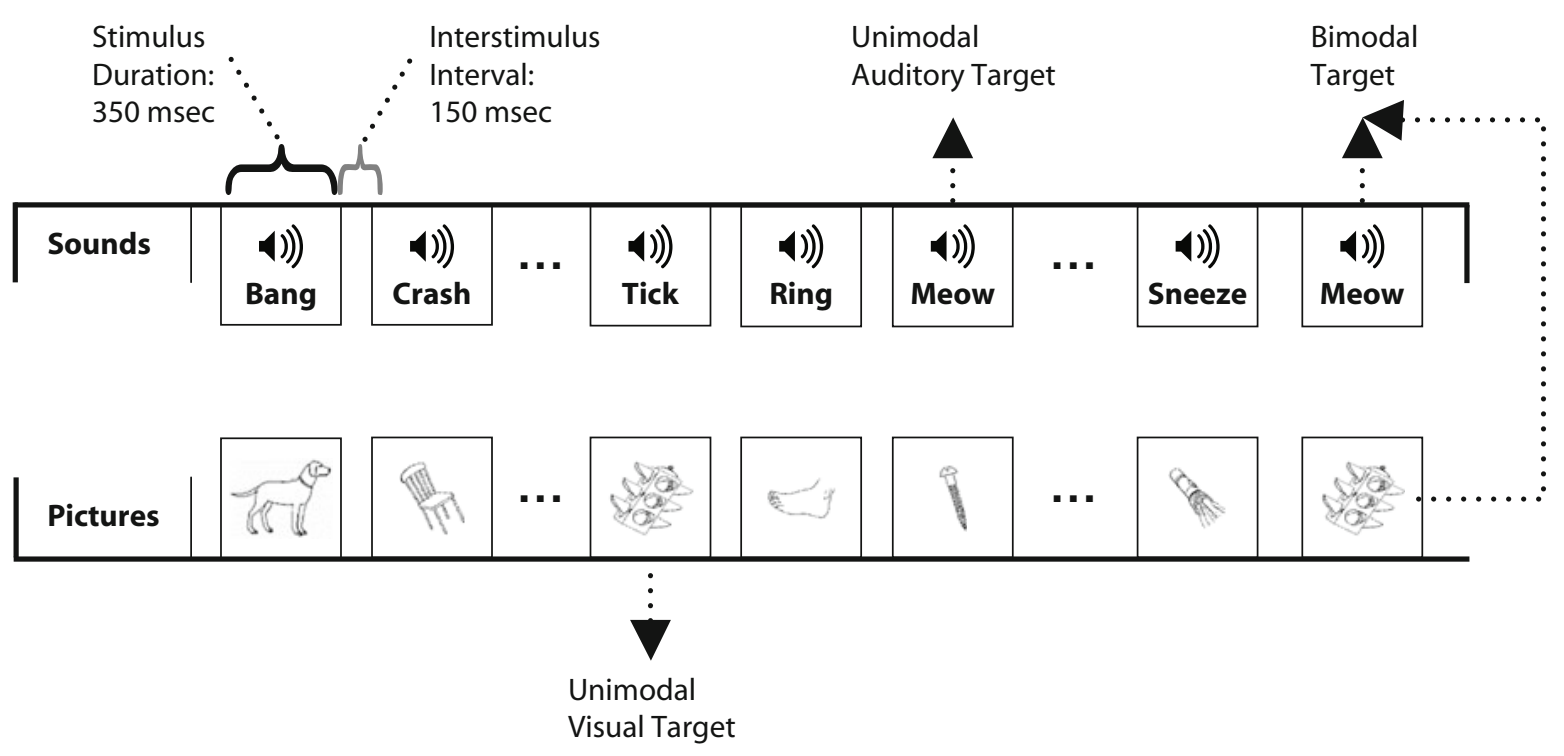

Figure 2. Illustration of the experimental design used in Experiments 2-6. Picture-sound pairs were presented simultaneously for $350 \mathrm{msec}$ and separated by $150 \mathrm{msec}$ of silence and a blank screen. The participants were instructed to monitor the two streams and to make one of three responses depending on whether (1) they saw a stoplight presented on the screen (unimodal visual target), (2) they heard a cat meowing (unimodal auditory target), or (3) they perceived both target stimuli at the same time (bimodal target).

cantly more rapidly to unimodal auditory and visual targets than to bimodal targets $[t(53)=7.0, p<.001$; and $t(53)=5.8, p<.001$, respectively]. Finally, auditory targets $(695 \mathrm{msec})$ were responded to slightly more rapidly than were visual targets $(721 \mathrm{msec})$, although the difference was only marginally significant $[t(53)=2.0, p=$ .055; see Figure 3B].

The results of Experiment 2 successfully extended the Colavita effect to a fundamentally different situation involving active searching among sequentially presented distractors - arguably a more complex and difficult task, statistically supported by the increase in errors to bimodal trials $[t(23)=7.7, p<.001]$.

In turn, this result raises the opportunity to further explore any potential role that attention may play in the visual dominance effect. However, before moving on, it is important to address some potential alternative explanations arising from the particular methodology used in Experiments 1 and 2 . In both experiments, bimodal targets occurred less frequently than either of the unimodal targets (see, for example, Quinlan \& Bailey, 1995; Spence, Nicholls, \& Driver, 2001), mirroring the distribution of stimuli used in all of Colavita's previous experiments, although the imbalance was slightly reduced here. This imbalance does not, in principle, affect the possible explanation of the results in terms of visual dominance, given that this explanation relies on the comparison restricted to the various types of errors made on bimodal trials only. It is nevertheless interesting to clarify whether the increase in RTs to bimodal targets, in comparison with unimodal targets, stems from the less frequent occurrence of bimodal trials or from the bimodal nature of the stimuli themselves. Experiment 3 was designed to address this issue, as well as the concern that there may be something special (or different) about the particular bimodal stimuli chosen to serve as the targets in Experiments 1 and 2-a stoplight for the visual stream and a cat meowing for the auditory stream). In Experiment 3, therefore, we tested participants' responses to three different bimodal targets, one of them being of lower frequency than either of the other two.

\section{EXPERIMENT 3}

\section{Method}

Participants. Thirty-six students from the University of Barcelona participated in Experiment 3 in exchange for course credit. All reported normal hearing and normal or corrected-to-normal vision, and were naive about the purpose of the experiment.

Apparatus, Materials, and Procedure. The materials and procedure were identical to those in Experiment 2. The only difference in Experiment 3 was that participants were instructed to respond to three different predefined bimodal targets. That is, rather than monitoring both streams (auditory and visual) for unimodal targets and the simultaneous occurrence of both targets (bimodal targets), participants were required to make one of three keypress responses to the occurrence of only bimodal targets. The three bimodal targets consisted of the pictures of a stoplight, an airplane, and a lightbulb respectively paired with the sounds of a cat, a sneeze, and a trumpet. The target frequency was counterbalanced across participants so that each target appeared in the low frequency $(20 \%)$ condition equally often across participants.

\section{Results and Discussion}

There were no differences in RT among the three different bimodal targets when presented in the 20\% lowfrequency condition, nor when they were presented in the $40 \%$ high-frequency condition. The data were therefore pooled across target pairings and analyzed in order to determine whether there were any differences in average 

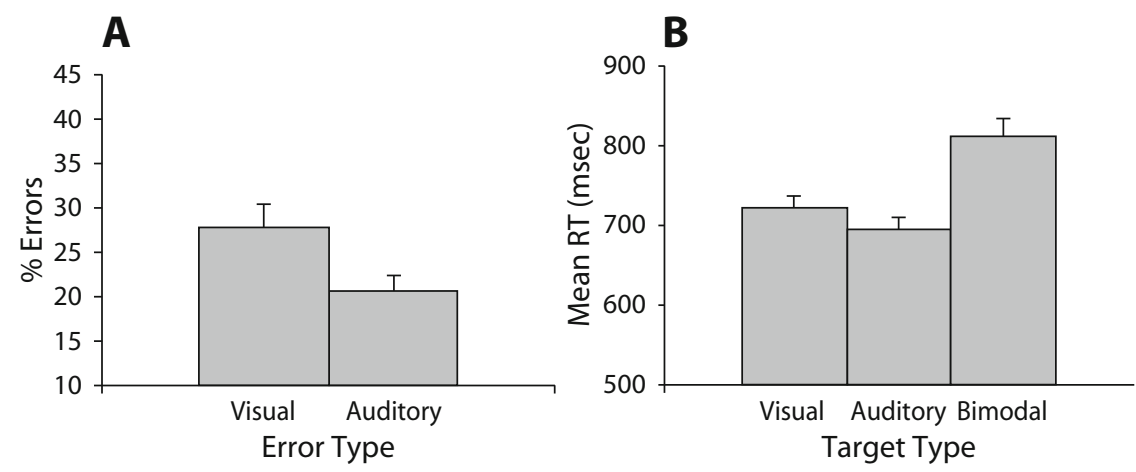

Figure 3. Results of Experiment 2. (A) The distribution of errors displayed as percentages of unimodal visual or unimodal auditory responses to bimodal trials. (B) Average RTs across the 3 target types (visual, auditory, and bimodal). The error bars represent the standard error of the mean for each condition.

RTs as a function of the different target proportions used. RTs for the low-frequency target $(658 \mathrm{msec})$ did not differ significantly from the RTs for high-frequency targets $(663$ and $644 \mathrm{msec})[t(35)=0.2, p=.843$, and $t(35)=$ $0.8, p=.449$, respectively]. Error rates for each target frequency did not differ $(10 \%$ and $7 \%$ for frequently occurring targets, $11 \%$ for infrequently occurring targets) and were comparable to the unimodal error rates reported in Experiment 2 ( $11 \%$ for unimodal auditory target trials, $10 \%$ for unimodal visual target trials).

The results of Experiment 3 suggest that neither the particular choice of experimental target stimuli used in Experiment 2, nor the relative frequency with which the bimodal targets were presented, had any sizeable effect on the response latency or accuracy measures reported. In addition to the finding that there was no statistical difference in the RTs as a function of whether the bimodal targets were presented with a low versus high frequency, it is important to note that no differences were observed among the three different versions of the bimodal target when they occurred with a low frequency (i.e., with a similar probability of occurrence as used in Experiments 1 and 2 ; all $p \mathrm{~s}>.5$ ).

Bimodal targets in Experiments 1 and 2 were always presented less frequently than unimodal targets in order to maintain the usual distribution of trials used in previous experimental studies of the Colavita effect (Colavita, 1974; Colavita et al., 1976; Colavita \& Weisberg, 1979) where the bimodal targets were presented much less frequently. For instance, Colavita et al. presented a total of just five bimodal targets to each of their participants (14\% of the trials). Experiment 3 showed that the increase in latency of the bimodal targets in Experiments 1 and 2 is not a result of the infrequency of bimodal target presentation. Yet, it could be argued that the response latencies to bimodal trials were slower in our first two experiments due to their relatively "unexpected" nature (i.e., their low frequency of occurrence in comparison with unimodal targets; whereas in Experiment 3 the comparisons were always between bimodal targets). In Experiment 4, we addressed this potential issue by equalizing the propor- tion of bimodal targets to those of each of the unimodal targets $-33 \%$ visual, $33 \%$ auditory, and 33\% bimodal.

\section{EXPERIMENT 4}

In Experiment 4, the proportion of each type of unimodal and bimodal target was equalized-33\% visual, $33 \%$ auditory, and 33\% bimodal. This modification addresses the concern that the infrequency of bimodal targets in Experiments 1 and 2 could explain the slower RTs to bimodal targets.

\section{Method}

Participants. Twenty-four students from the University of Barcelona participated in Experiment 4 in exchange for course credit. All reported normal hearing and normal or corrected-to-normal vision, and were naive about the purpose of the experiment.

Apparatus, Materials, and Procedure. The materials and procedure were identical to those used in Experiment 2, the only differences being that the frequency of bimodal targets was increased from $20 \%$ to $33 \%$, and the frequency of unimodal targets was reduced from $40 \%$ to $33 \%$, yielding 44 unimodal visual targets, 44 unimodal auditory targets, and 44 bimodal targets.

\section{Results and Discussion}

The RT data and error data from the bimodal target trials were analyzed just as in Experiment 2. False alarm rates for responses to nontarget trials were less than $0.01 \%$. Participants missed $13 \%$ of the unimodal auditory target trials, $14 \%$ of the unimodal visual target trials, and failed to respond in $0.2 \%$ of the bimodal target trials. Errors were frequent on the bimodal target trials $(37.8 \%$ error rate overall) and, critically, participants made erroneous unimodal visual responses significantly more often than they did unimodal auditory responses $[23.5 \%$ vs. $13.8 \%$; $t(23)=2.1, p=.051$; see Figure 4A]. In terms of the RT data, participants responded significantly more rapidly to unimodal auditory or visual targets than to bimodal targets $[t(23)=2.5, p=.019$; and $t(23)=2.9, p=.009$, respectively]. Finally, response latencies to auditory targets (713 msec) and visual targets $(711 \mathrm{msec})$ did not differ significantly $[t(23)=0.1, p=.938$; see Figure $4 \mathrm{~B}]$. 

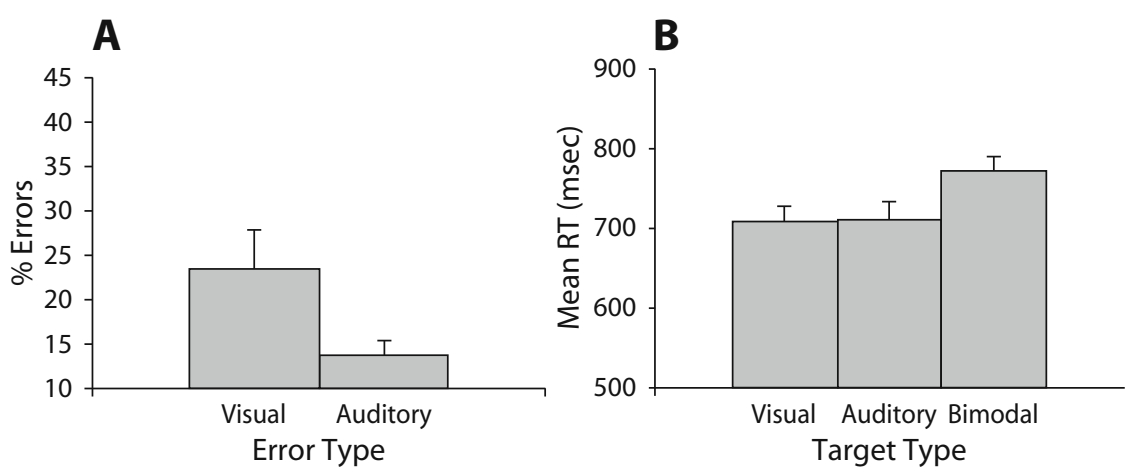

Figure 4. Results of Experiment 4. (A) The distribution of errors displayed as percentages of unimodal visual or unimodal auditory responses to bimodal trials. (B) Average RTs across the 3 target types (visual, auditory, and bimodal). The error bars represent the standard error of the mean for each condition.

The results of Experiment 4 are interesting because they indicate that, in contrast with the generalized tendency of making the bimodal target less frequent-and, therefore, to some extent, unexpected - in all previous studies, the Colavita effect (1974) persists even when bimodal targets are presented as frequently as either of the unimodal targets. Thus, it is difficult to claim that either the increased latency to bimodal targets or the visual dominance in errors, as observed in Experiments 1 and 2, is somehow related to presenting the bimodal targets at a lower frequency. On bimodal trials, the participants erroneously responded with the visual response key significantly more often than with the auditory response key. Furthermore, just as in Colavita's studies, no significant difference in response latency to unimodal auditory and visual targets was found when mixing them within the same block of trials (except in Experiment 2), where RTs to auditory targets were marginally faster than RTs to visual targets. This other possible manifestation (faster RTs to unimodal auditory targets than to unimodal visual targets) has been observed when targets are not interspersed in the same block (see Colavita, 1974).

\section{EXPERIMENT 5}

Experiments 1-4 successfully addressed the goal of extending the Colavita (1974) visual dominance effect to more complex stimulus arrays and situations. At this point, we adapted our paradigm to address the second goal of the present study - namely, to discover what role, if any, attention plays in the Colavita visual dominance effect.

Posner et al. (1976) claimed that visual dominance was a result of people having an attentional bias toward the visual modality in order to compensate for the poor alerting qualities of vision. However, in apparent contradiction with this attentional account, further experimentation by Colavita (1974, Experiment 2) suggested that the visual prepotency effect might actually occur at a sensory level, prior to the engagement of attention. Colavita found that the visual dominance effect described above was still observed, even after the experimental stimuli were adjusted so that the auditory stimuli appeared to be (subjectively) twice as intense as the visual stimuli (i.e., adjusting the loudness of a $4000-\mathrm{Hz}$ tone until it was judged to be twice as intense as a 50-footcandle light). Under such conditions, participants still made unimodal visual responses on 97 out of 122 bimodal trials. ${ }^{3}$

In a subsequent experiment (Colavita, 1974, Experiment 4), participants were informed about the presence of bimodal target trials prior to the start of the experiment and were explicitly instructed to respond to the auditory tone on those trials. Despite this instruction, participants once again responded to the light on 36 of the 60 bimodal trials. Thus, it appears that, while the instructional manipulation reduced the magnitude of the visual dominance effect somewhat - in terms of lowering the preponderance of unimodal visual responses on bimodal stimulation trials - it was not possible to abolish it completely, prompting Colavita to claim that visual dominance may occur regardless of the allocation of a participant's attention. However, it should be noted that values of statistical significance were not provided in Colavita's study, nor were these data compared with any kind of baseline (e.g., the same experiment, but without the instructional manipulation). Furthermore, due to experimenter expectancy effects (see, e.g., Intons-Peterson, 1983; Orne, 1962; Pierce, 1908) and the lack of a third response key, it is possible that Colavita's experiments failed to adequately measure the potential role of attention in affecting the measure and apparent evidence of visual dominance.

In line with the notion that visual dominance cannot be explained solely by attentional biases, Colavita et al. (1976) also failed to abolish the Colavita effect by diverting the direction of participants' gaze (their overt attention) away from the location where the visual stimuli were presented and toward the source of auditory stimulation. The authors separated the sources of the auditory and visual stimuli by $25 \mathrm{~cm}$ and, in trials where both the light and tone were presented simultaneously, instructed participants to respond to whichever stimulus they became aware of first. When the participants were required to fixate midway between the auditory and visual stimuli, $84 \%$ of the bimodal trials 
yielded visual responses, thus replicating the basic Colavita visual dominance effect. When participants fixated on the loudspeaker used to present the sounds instead (i.e., away from the light flash), the visual dominance effect was only slightly diminished (78\% light responses, although the statistical significance of this difference was not reported).

In summary, it appears that the only experimental manipulation that successfully reduced the magnitude of the Colavita visual dominance effect was the explicit instruction given to the participant to respond to the auditory stimulus on bimodal trials, a manipulation that could have introduced a response bias. Note, however, that even while using such a strong manipulation, participants still made visual responses on more than half of the bimodal trials. These results were interpreted as supporting the notion that visual dominance may reflect a mechanism that is little influenced by endogenous attention, in contrast with the claims made by Posner et al. (1976; see also Spence, Shore, \& Klein, 2001). Experiments 5 and 6 were designed to address these conflicting hypotheses by using the paradigm introduced in Experiment 2 to investigate whether the distribution of attention to a sensory modality (audition or vision) would affect visual dominance.

If the attentional account of visual sensory dominance were to prove correct, faster response latencies to auditory targets and a reduction in visual responses to bimodal targets should be observed when attention is directed toward the auditory modality. Similarly, when attention is directed toward the visual modality, the attentional account of visual dominance would predict an increase in visual errors on bimodal trials and faster response latencies to visual targets. In contrast, a purely sensory account of visual dominance would presumably have to predict a bias of equal magnitude for erroneous visual responses to bimodal targets, regardless of the direction of a participant's attention, just as reported by Colavita (1974).

We tested this prediction in Experiment 5; we manipulated the direction of participants' attention toward either the auditory or visual modality by altering the relative probability of occurrence of unimodal auditory and visual targets (see Quinlan, 2000). This manipulation should bias the direction of attention to the more frequent modality. It should also be noted that increasing the frequency of targets in one modality also introduces a potential bias in response mechanisms: Increased frequency in one modality may simply prime the particular responses associated with the more frequent stimuli, thereby confusing any modulation in visual dominance for attentional reasons. However, this seemed to represent the strongest possible experimental manipulation to start with (see also Colavita, 1974, Experiment 4). If changes in the level of visual dominance were not observed, this would make any future experimentation investigating the role of endogenous attention in visual dominance useless, and would provide strong evidence for basing the causes of the Colavita effect in purely sensory mechanisms.

\section{Method}

Participants. Thirty-six students from the University of Barcelona took part in Experiment 5 in exchange for course credit. All reported normal hearing and normal or corrected-to-normal vision, and all were naive about the purpose of the study.

Apparatus, Materials, and Procedure. These were identical to those in Experiment 2, the only difference being the relative proportion of targets presented in each sensory modality. For the auditory biased group, the frequency of the unimodal auditory targets was increased to $60 \%$, and the frequency of the unimodal visual targets was reduced to $20 \%$. The reverse was true of the visually biased group. Note that the ratio of unimodal to bimodal targets remained the same as in Experiment 2. Each participant was tested in only one of the conditions (auditory or visual). The participants were not informed about the manipulation of target probability (cf. Quinlan \& Bailey, 1995).

\section{Results and Discussion}

Auditory biased group. Errors on bimodal targets (41.9\% error rate overall) were divided fairly evenly between visual and auditory responses (18.7\% vs. $21.2 \%)$ $[t(17)=0.6, p=.575$; see Figure 5A]. Participants responded significantly more rapidly to auditory targets $(661 \mathrm{msec})$ than to either visual targets $(804 \mathrm{msec})$ or bimodal targets $(814 \mathrm{msec})[t(17)=5.3, p<.001 ; t(17)=$ $8.75, p<.001$, respectively; see Figure 5B]. Response times to bimodal and visual targets did not differ significantly $[t(17)=0.3, p=.749]$. The false alarm rates for responses to nontarget trials were less than $0.2 \%$. Participants missed $10 \%$ of the auditory targets, $18 \%$ of the visual targets, and $1 \%$ of the bimodal targets.

Visual biased group. Errors on bimodal trials (55.5\% error rate overall) were strongly biased toward visual responses $(33.8 \%$ vs. $21.5 \%)[t(17)=2.3, p=.037$; see Figure $5 \mathrm{C}]$. The participants responded significantly more rapidly to visual targets $(618 \mathrm{msec})$ than to either auditory targets $(723 \mathrm{msec})$ or bimodal targets $(727 \mathrm{msec})[t(17)=$ $3.3, p=.004$; and $t(17)=3.9, p=.001$, respectively; see Figure 5D]. Response latencies to bimodal targets were not significantly different from RTs to auditory targets $[t(17)=0.1, p=.937]$. The false alarm rates for responses to nontarget trials were less than $0.01 \%$. Participants missed $15 \%$ of the auditory targets, $10 \%$ of the visual targets, and $0.3 \%$ of the bimodal targets.

Of particular interest, the overall error rate for bimodal trials was much greater in the visual attention group in comparison with the auditory attention group $[55.5 \%$ vs. $41.9 \% ; t(17)=2.4, p=.026]$. This difference can best be explained by the increase in the visual dominance effect (i.e., in Colavita errors) in bimodal trials of the visual biased group. Note that the percentage of auditory-based errors was the same for both attention conditions; therefore, the increase in visually based errors should also be reflected in the overall error rate for bimodal trials.

The data from the auditory bias group suggest that Colavita's (1974) visual dominance effect can be significantly reduced by biasing the observers' focus of attention toward the auditory modality (i.e., by increasing the probability of auditory targets relative to that of visual targets). The data from both conditions of Experiment 5 were pooled in order to explore any effect of bias on response latency. An ANOVA with the between-participants factor frequent modality (auditory vs. visual) and the within-participants factor target modality (unimodal vi- 

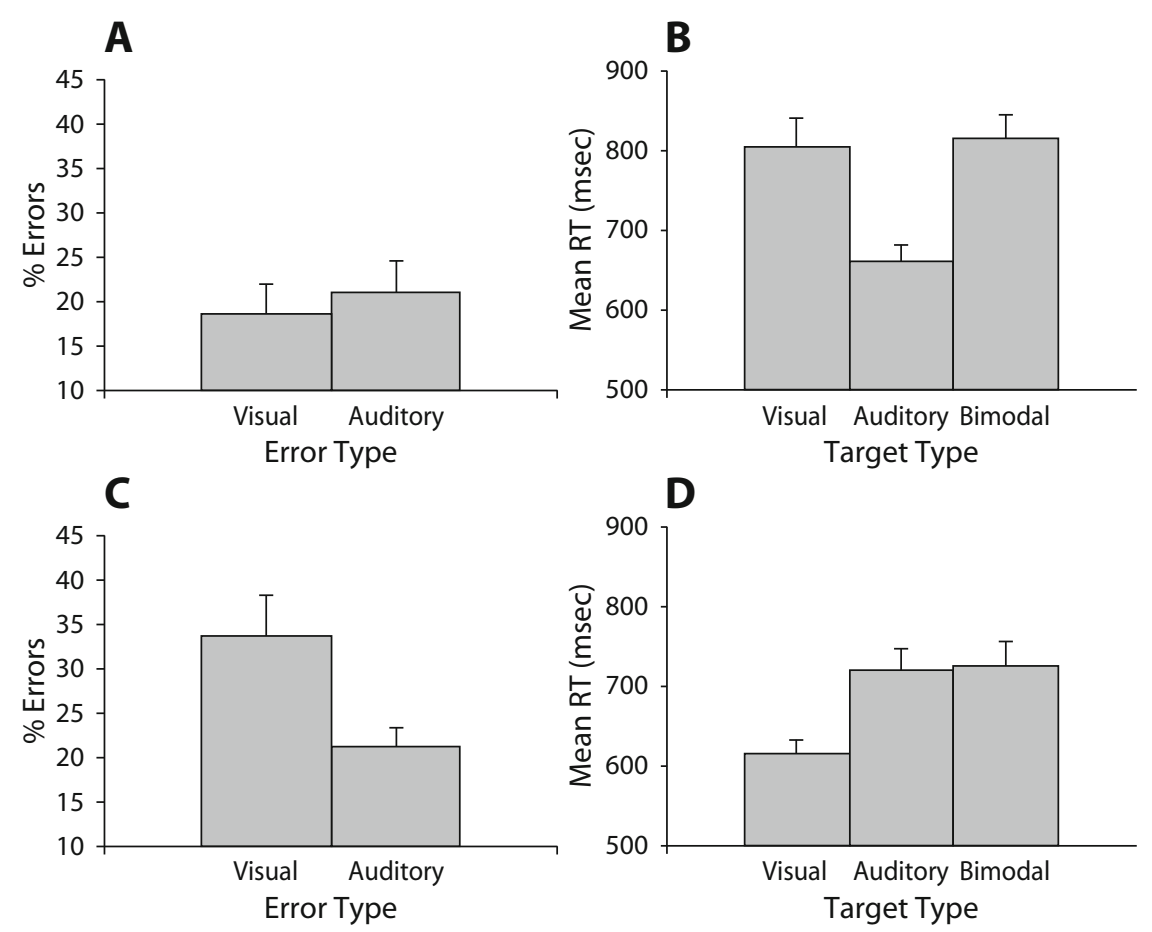

Figure 5. Results of Experiment 5. Panels $A$ and $C$ represent the distribution of errors in terms of the percentages of unimodal visual or unimodal auditory responses to bimodal trials. Panels $B$ and D represent the average RTs from the 3 target types (visual, auditory, and bimodal). The error bars represent the standard error of the mean for each condition. Panels $A$ and $B$ represent data from the auditory bias condition, whereas panels $C$ and $D$ represent data from the visual bias condition.

sual vs. unimodal auditory) was conducted. This revealed no main effect of target modality $[F(1,72)=0.53, p=$ .47], but a significant effect of frequent modality was observed $[F(1,72)=5.7, p=.02]$. This latter result shows that the direction of the bias to a specific sensory modality had a significant effect on response latencies. The interaction between frequent modality and target modality was also significant $[F(1,72)=22.9, p<.001]$. Further $t$ tests revealed that increases in target frequency for each modality created a decrease in unimodal RTs to the target with a higher frequency, although this effect was only marginally significant in the auditory modality, despite a 62 -msec RT difference $[t(17)=1.9, p=.074]$, and highly significant for visual targets $[t(17)=4.6, p<.001]$. It is interesting to note here that, despite the large advantage in RTs for auditory versus visual targets in the auditory bias condition (143 msec faster), the percentage of errors was equally divided between visual and auditory responses on the bimodal trials $(18.7 \%$ vs. $21.2 \%$; nonsignificant difference). On the other hand, the results of the visual bias condition showed an exaggerated visual dominance effect on bimodal trials ( $12.3 \%$ difference in favor of visual errors) in addition to a visual advantage in the speed of responses to visual trials $(105 \mathrm{msec})$. Interestingly, it appears that the frequency manipulation only affected the visual errors on bimodal trials. That is, auditory errors remained relatively constant regardless of the proportion of auditory targets presented (20.5\% in Experiment 2;
$21.2 \%$ in Experiment 5, auditory bias group; and 21.5\% in Experiment 5, visual bias group). This pattern of results corresponds well with the idea that attentional manipulations might have a major impact on visual processing, just as Posner et al. (1976) originally claimed. One possible explanation for the fact that a complete reversal in errors after bimodal trials was not observed is that there may be some kind of residual bias toward the visual modality (see Battaglia et al., 2003; Spence, Shore, \& Klein, 2001).

The results of Experiment 5 are revealing: They show that, by introducing a strong bias (attentional and response-related) toward a sensory modality, it is possible to manipulate the size of the Colavita visual dominance effect, although not to reverse it completely. However, it is now necessary to investigate whether a purely attentional component of the manipulation (i.e., without altering the relative probabilities with which participants have to make the various responses) can also effectively modulate the Colavita effect. This was precisely the goal of our next, and final, experiment.

\section{EXPERIMENT 6}

We addressed the role of attention in visual dominance by manipulating the perceptual load within each modality individually. According to the perceptual load theory (Lavie, 2005), the processing of task-irrelevant information can be reduced considerably if the processing de- 
mands required by the task-relevant information are sufficiently high. That is, if the perceptual load is relatively low, extra attentional resources will be available to process distracting stimuli in the same sensory modality (Rees, Frith, \& Lavie, 2001), and they can therefore exert an interference effect on the task-relevant processes. However, if the perceptual load is increased, the available attentional resources will be fully utilized by the task at hand, and thus no available resources will remain to process other distracting stimuli in the same modality. Note that the effects of load manipulation in one modality on the processing of information in another modality tend to be smaller (Sinnett, Costa, \& Soto-Faraco, 2006) or even completely absent under some conditions (Rees et al., 2001), thereby suggesting that it is possible to manipulate attentional load in a specific modality without affecting perception levels in another modality. This logic can be applied to our paradigm in order to disentangle the role of attention in modulating the visual dominance effect. By manipulating the variability among irrelevant distractors in each modality stream (visual or auditory), we can manipulate the perceptual load in either modality, and therefore the amount of attentional resources that are available to process the targets in that modality. Specifically, if there are fewer types of distracting stimuli in the visual stream, more visual resources should be available; thus, the level of visual dominance, as represented by visually based errors to bimodal trials, should increase. The reverse is expected if the variability of auditory distractors is reduced - that is, less visual dominance - as there should be more attentional resources available to process the residual auditory stimuli.

\section{Method}

Participants. Thirty-six students from the University of Barcelona took part in Experiment 6 in exchange for course credit. All reported normal hearing and normal or corrected-to-normal vision, and were naive about the purpose of the study.

Apparatus, Materials, and Procedure. These were exactly the same as in Experiment 2, the only difference being the number of distracting drawings or sounds presented in each stream. Specifically, to free up visual attentional resources in the visual condition, the number of distracting drawings (i.e., drawings other than the stoplight) was reduced from 49 to 3 . By comparison, in the auditory condition, reducing the number of distracting sounds (i.e., sounds other than the cat meowing) from 49 to 3 should free up auditory attentional resources. As in Experiment 5, each participant was tested in only one of the conditions, either auditory or visual.

\section{Results and Discussion}

Low auditory load group. The overall error rate for bimodal targets was $38.9 \%$. There were more visually based errors after bimodal targets than auditory based errors $[26.5 \%$ vs. $11.6 \% ; t(17)=4.6, p<.001$; see Figure $6 \mathrm{~A}]$. The participants responded significantly more rapidly to visual targets $(632 \mathrm{msec})$ than to bimodal targets $[702 \mathrm{msec} ; t(17)=2.4, p=.031]$. Responses to auditory targets $(645 \mathrm{msec})$ were slightly faster than responses to bimodal targets, and not significantly different from response latencies to visual targets $[t(17)=1.9, p=.078$; $t(17)=0.7, p=.496$, respectively; see Figure 6B]. False alarm rates for responses to nontarget trials were less than $0.01 \%$. Participants missed $10 \%$ of the auditory targets, $8 \%$ of the visual targets, and $0.8 \%$ of the bimodal targets.

Low visual load group. Errors on bimodal trials (55\% error rate overall) were strongly biased toward visual responses $(39.1 \%$ vs. $14.1 \%)[t(17)=7, p<.001$; see Figure $6 \mathrm{C}]$. The participants responded significantly more rapidly to visual $(667 \mathrm{msec})$ or auditory $(674 \mathrm{msec})$ targets than to bimodal targets $(759 \mathrm{msec})[t(17)=3.9$, $p=.001$, and $t(17)=2.9, p=.01$, respectively; see Figure $6 \mathrm{D}]$. There was no significant difference between the response latencies to visual and auditory targets $[t(17)=$ $0.4, p=.699]$. False alarm rates for responses to nontarget trials were less than $0.2 \%$. Participants missed $18 \%$ of the auditory targets, $11 \%$ of the visual targets, and $0.8 \%$ of the bimodal targets.

The results of Experiment 6 provide empirical support for the claim that the Colavita (1974) visual dominance effect can be affected by attentional manipulations. First, reducing the perceptual load in the auditory modality produced less visual dominance than did reducing the perceptual load in the visual modality $[26.5 \%$ vs. $39.1 \%$ visually based errors; $t(17)=2.4, p=.03$ ]. When subtracting the percentage of auditory-based errors from the percentage of visually based errors in both conditions of this experiment, the difference is much larger (i.e., the visual dominance effect is greater) when there are more visual attentional resources available $[25.0 \%$ vs. $14.9 \%$; $F(1,34)=4.4, p=.044]$. Thus, it appears that the magnitude of visual dominance can be significantly affected by attentional manipulations, at least when driven by changes in perceptual load.

Although the results of Experiments 5 and 6 converge on the same conclusion, there are two main differences between them that deserve to be mentioned. First, the significant effects that the attentional manipulations in Experiment 5 had on response latency were not observed in Experiment 6. The RT data from both conditions of Experiment 6 were pooled, and an ANOVA with the betweenparticipants factor of perceptual load modality (auditory vs. visual) and the within-participants factor of target modality (unimodal visual vs. unimodal auditory) revealed no main effect of target modality $[F(1,72)=0.2, p=.66]$, nor of perceptual load modality $[F(1,72)=2.2, p=.143]$. The interaction between perceptual load modality and target modality also failed to reach statistical significance $[F(1,72)=0.01, p=.925]$. It seems likely therefore that the increased proportions of targets in a given modality in Experiment 5 caused a response-related advantage for the modality that was more frequently responded to, a factor that was controlled for in Experiment 6 .

The second major difference between the results of Experiments 5 and 6 is that visual dominance (indexed by the imbalance in the number of visual and auditory errors to bimodal trials) in the latter experiment was not eliminated completely in the auditory condition, as it had been in Experiment 5. Once again, it is likely that the increased proportion of auditory targets in Experiment 5 could have created a response bias and, therefore, correspondingly 

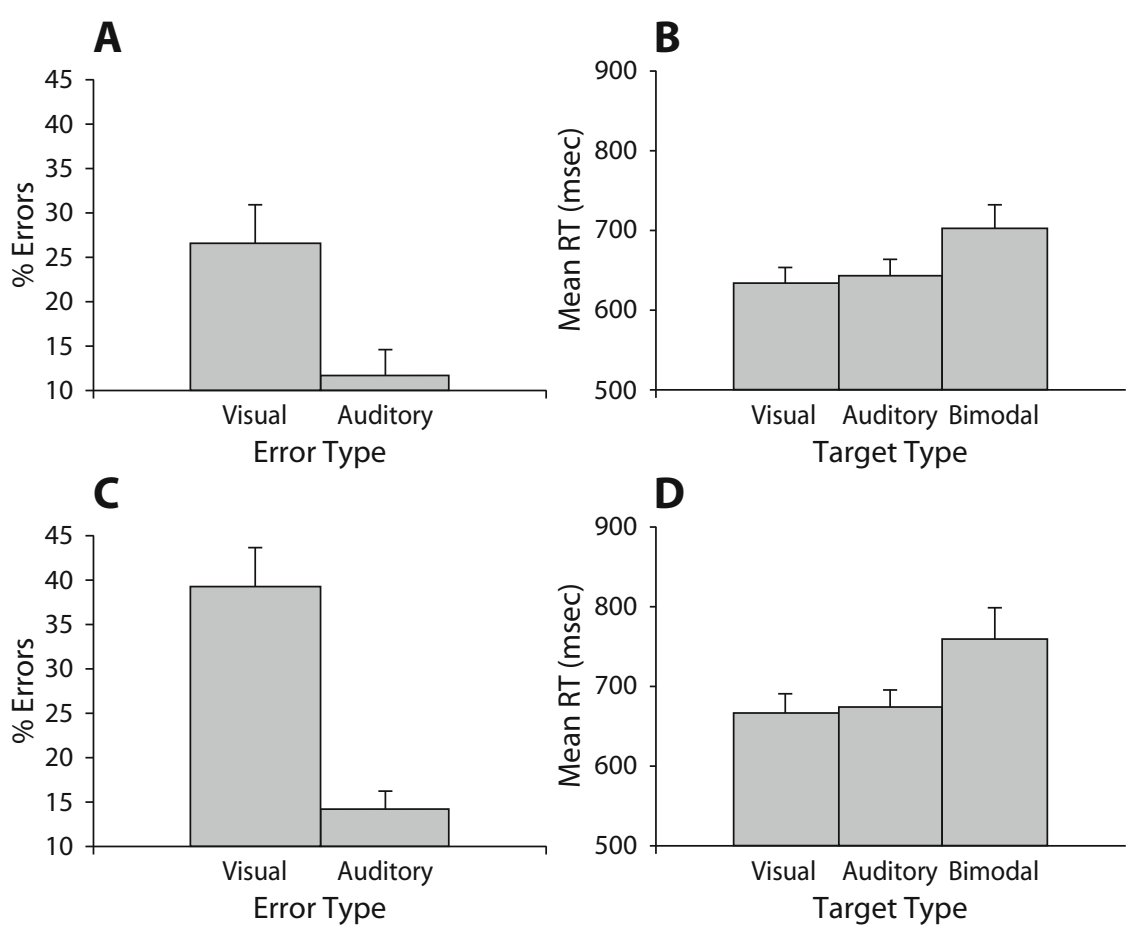

Figure 6. Results of Experiment 6. Panels $A$ and $C$ represent the distribution of errors displayed as percentages of unimodal visual or unimodal auditory responses to bimodal trials. Panels B and D represent the average RTs from the 3 target types (visual, auditory, and bimodal). The error bars represent the standard error of the mean for each condition. Panels $A$ and $B$ represent data from the auditory attention condition, whereas panels $C$ and $D$ represent data from the visual attention condition.

decreased the magnitude of the visual dominance effect reported. The experimental modifications introduced in Experiment 6 eliminated the response bias component. Combined, the results of these two experiments suggest that visual dominance is susceptible to manipulations of attention, although it appears that visual dominance cannot be entirely reversed (i.e., to create auditory dominance), despite the strong response and attention manipulation introduced in Experiment 5, nor eliminated (i.e., cancelling out the imbalance between visual and auditory errors) when manipulating perceptual load to free up auditory attentional resources (Experiment 6).

\section{GENERAL DISCUSSION}

Many aspects of our everyday lives involve the processing of multisensory events in highly complex environments (see Basil, 1994). The notion that vision is the dominant sensory modality for humans appears frequently in the literature (see, e.g., Colavita, 1974; Colavita et al., 1976; Posner et al., 1976; Rock \& Harris, 1967; Rock \& Victor, 1964; Spence, Shore, \& Klein, 2001). However, the majority of the studies on which such claims have been based have only tested simple stimuli, such as light flashes and tones, in contexts devoid of any complexity. The experiments reported here represent the only extension of the Colavita visual dominance effect to study responses to more complex stimulation conditions.
How can it be that the participants in the present study responded just as rapidly to the unimodal auditory and visual stimuli (even faster to auditory targets in Experiment 2), but nevertheless responded more frequently to only the visual target than to only the auditory target on bimodal trials (Experiments 1, 2, 4, 5 [visual condition], and Experiment 6)? One possible account for this paradoxical (but clearly replicable) pattern of results (see also Colavita, 1974; Colavita et al., 1976; Colavita \& Weisberg, 1979; Kristofferson, 1967 ) is based on the notion of attentional capture. It is likely that, if only one target stimulus (auditory or visual) is present, then attention will rapidly be directed to that stimulus. It is also known that the brain's processing of an auditory event typically takes place somewhat earlier in time (from stimulus onset) than for a visual event (Giard \& Peronnet, 1999), thereby potentially explaining the advantage in RTs for unimodal auditory stimuli when presented in different blocks (see also Spence \& Squire, 2003). If, however, an auditory and a visual target are presented at approximately the same time (as in the bimodal trials of the present study), then attention may be captured preferentially by the visual stimulus and hence take a lead in processing (see Bald, Berrien, Price, \& Sprague, 1942; Whipple, Sanford, \& Colegrove, 1899, on this point). This idea fits with Broadbent's (1958; see also Lachter, Forster, \& Ruthruff, 2004) channelswitching model of attention, which suggests that in the case of bimodal stimulation, one channel (in this case, the visual modality) is sampled first, prior to the processing of stimuli 
in the second channel. Viewed in this way, our results could be taken to suggest that, under conditions of divided attention, the visual channel is sampled before, or possibly more frequently than, the auditory channel. However, because this difference in sampling rate (or bias) may be attentional in nature, it can be manipulated by focusing attention on one sensory modality or another, as seen in investigations of prior entry (see Spence, Shore, \& Klein, 2001; Titchener, 1908) and in the results of Experiments 5 and 6 (see Cooper, 1998, for a similar model).

It should be noted that the attentional manipulations used in Experiments 5 and 6 were unable to completely reverse the visual dominance effect (i.e., to create auditory dominance); therefore, it cannot be concluded that visual dominance is based entirely on attentional mechanisms. The contribution of the present study is that, after controlling for the methodological shortcomings typically present in previous research (e.g., Colavita, 1974; Egeth \& Sager, 1977), the magnitude of visual dominance can be significantly modified by attentional manipulations.

An interesting trend observed across all of the experiments reported in the present study was that bimodal targets were rarely missed (all experiments, $<2 \%$ ), whereas the failure to respond to unimodal targets was, relatively speaking, much more frequent $(M=11.5 \%, S D=4.5 \%)$. This would suggest at least some type of enhancement for bimodal presentations. Indeed, it has been shown that visual perception is enhanced when the presentation of visual stimuli is combined with auditory events (see, e.g., Frassinetti, Bolognini, \& Làdavas, 2002; McDonald, TederSälejärvi, \& Hillyard, 2000). Whether this enhancement is purely statistical in nature - the base probability of completely missing a bimodal target being logically smaller than missing a unimodal target - or arises from a nonlinear interaction between audition and vision is yet to be seen.

Colavita and his colleagues (Colavita, 1974; Colavita et al., 1976; Colavita \& Weisberg, 1979) had very limited success in weakening the visual dominance effect through various task manipulations, such as reducing the tendency of participants to make a visual response on bimodal trials, leading to the conclusion that visual dominance does not reflect an attentional phenomenon. However, this argument holds little weight, as one could easily argue that the visual stimulus simply exogenously captured attention before the auditory stimulus could (see Rodway, 2005; Turatto, Benso, Galfano, \& Umiltà, 2002). When perceptual load is low in a given modality, modality-specific attentional resources become available to process the target, having the effect of modulating the magnitude of visual dominance. Therefore, we argue that the present results support the notion that at least some component of the visual dominance effect is attentional in nature (see Quinlan, 2000, for a related example). However, based on the results of Experiment 5, it should be noted that, in order to reduce the magnitude of the visual dominance effect, a strong bias to attend toward - and to respond toward - the auditory modality must be induced. That is, even when greatly increasing the proportion of auditory targets so that RTs to unimodal auditory targets are much faster than to unimodal visual targets, participants exhibited only a very small (nonsignificant) bias toward making more erroneous unimodal auditory than unimodal visual responses after bimodal trials. The fact that a complete reversal in errors under auditory attention conditions was not observed suggests that visual dominance may also involve residual biases toward the visual modality (see also Battaglia et al., 2003). Whether this bias can be attributed to sensory or attentional mechanisms (based on exogenous mechanisms) is difficult to determine at present.

The neurological underpinnings of sensory perception have been discussed by Driver and Frackowiak (2001) in the context of a "biased competition" model of attention (see also Desimone \& Duncan, 1995). Driver and Frackowiak argued that multiple concurrent stimulus inputs will "compete" to drive neurons where these inputs meet - that is, in the large receptive fields at higher levels of the visual system, or in multisensory areas for the case of competing concurrent stimuli presented in distinct sensory modalities (see Macaluso \& Driver, 2001). While such competition may initially be played out within separate contexts, proponents of the biased competition model argue that the "winner" of such a competition in one context might tend to become dominant in other contexts by boosting the activation of the associated neural populations. As a result, activity in a network of different areas may come to converge on a common "winner" (see Macaluso \& Driver, 2001). This biased competition model could help to account for the tendency of participants in the present study to base their responses on the visual input.

\section{AUTHOR NOTE}

This research was supported by grants from the Ministerio de Ciencia y Tecnología (TIN2004-04363-C03-02) to S.S.-F. and the Oxford McDonnell Pew Center for Cognitive Neuroscience to S.S.-F. and C.S. We thank three anonymous reviewers for their helpful comments on a previous draft of this article. Correspondence concerning this article should be addressed to S. Sinnett, Brain and Attention Research Laboratory, University of British Columbia, 2136 West Mall, Vancouver, BC, V6T 1Z4 Canada (e-mail: ss@psych.ubc.ca).

\section{REFERENCES}

Bald, L., Berrien, F. K., Price, J. B., \& Sprague, R. O. (1942). Errors in perceiving the temporal order of auditory and visual stimuli. Journal of Applied Psychology, 26, 382-388.

BASIL, M. D. (1994). Multiple resource theory: I. Application to television viewing. Communication Research, 21, 177-207.

Battaglia, P. W., Jacobs, R. A., \& Aslin, R. N. (2003). Bayesian integration of visual and auditory signals for spatial localization. Journal of the Optical Society of America A, 20, 1391-1397.

Broadbent, D. E. (1958). Perception and communication. Elmsford, NJ: Pergamon.

Colavita, F. B. (1974). Human sensory dominance. Perception \& Psychophysics, 16, 409-412.

Colavita, F. B., Tomko, R., \& Weisberg, D. (1976). Visual prepotency and eye orientation. Bulletin of the Psychonomic Society, 8, 25-26.

Colavita, F. B., \& Weisberg, D. (1979). A further investigation of visual dominance. Perception \& Psychophysics, 25, 345-347.

Cooper, R. (1998). Visual dominance and the control of action. In M. A. Gernsbacher \& S. J. Derry (Eds.), Proceedings of the 20th Annual Conference of the Cognitive Science Society (pp. 250-255). Mahwah, NJ: Erlbaum.

Desimone, R., \& Duncan, J. (1995). Neural mechanisms of selective visual attention. Annual Review of Neuroscience, 18, 193-222.

Driver, J., \& Frackowiak, R. S. J. (2001). Neurobiological measures of human selective attention. Neuropsychologia, 39, 1257-1262. 
EgEth, H. E., \& SAGER, L. C. (1977). On the locus of visual dominance. Perception \& Psychophysics, 22, 77-86.

Eimer, M. (2000). The time course of spatial orienting elicited by central and peripheral cues: Evidence from event-related brain potentials. Biological Psychology, 53, 253-258.

ERNST, M. O., \& BANKS, M. S. (2002). Humans integrate visual and haptic information in a statistically optimal fashion. Nature, 415, 429-433.

ERnst, M. O., BANKs, M. S., \& BÜLthofF, H. H. (2000). Touch can change visual slant perception. Nature Neuroscience, 3, 69-73.

Ernst, M. O., \& BülthofF, H. H. (2004). Merging the senses into a robust percept. Trends in Cognitive Sciences, 8, 162-169.

Foree, D. D., \& LoLordo, V. M. (1973). Attention in the pigeon: Differential effects of food-getting versus shock-avoidance procedures. Journal of Comparative \& Physiological Psychology, 85, 551-558.

FrassinetTi, F., Bolognini, N., \& Làdavas, E. (2002). Enhancement of visual perception by crossmodal visuo-auditory interaction. Experimental Brain Research, 147, 332-343.

Giard, M. H., \& Peronnet, F. (1999). Auditory-visual integration during multimodal object recognition in humans: A behavioral and electrophysiological study. Journal of Cognitive Neuroscience, 11, 473-490.

GiLbert, R. M. (1969). Discrimination learning? In R. M. Gilbert \& N. S. Sutherland (Eds.), Animal discrimination learning (pp. 455489). New York: Academic Press.

Hohnsbein, J., Falkenstein, M., Hoormann, J., \& Blanke, L. (1991). Effects of crossmodal divided attention on late ERP components: I. Simple and choice reaction tasks. Electroencephalography \& Clinical Neurophysiology, 78, 438-446.

Intons-Peterson, M. J. (1983). Imagery paradigms: How vulnerable are they to experimenters' expectations? Journal of Experimental Psychology: Human Perception \& Performance, 9, 394-412.

KANwisher, N. (2001). Faces and places: Of central (and peripheral) interest. Nature Neuroscience, 4, 455-456.

KLEIN, R. M. (1977). Attention and visual dominance: A chronometric analysis. Journal of Experimental Psychology: Human Perception \& Performance, 3, 365-378.

Kraemer, P. J., \& RoberTs, W. A. (1985). Short-term memory for simultaneously presented visual and auditory signals in the pigeon. Journal of Experimental Psychology: Animal Behavior Processes, 11, 137-151.

Kristofferson, A. B. (1967). Attention and psychophysical time. Acta Psychologica, 27, 93-100.

Lachter, J., Forster, K. I., \& Ruthruff, E. (2004). Forty-five years after Broadbent (1958): Still no identification without attention. Psychological Review, 111, 880-913.

LAVIE, N. (2005). Distracted and confused?: Selective attention under load. Trends in Cognitive Sciences, 9, 75-82.

Lederman, S. J., \& Аввотt, S. G. (1981). Texture perception: Studies of intersensory organization using a discrepancy paradigm, and visual versus tactual psychophysics. Journal of Experimental Psychology: Human Perception \& Performance, 7, 902-915.

Macaluso, E., \& Driver, J. (2001). Spatial attention and crossmodal interactions between vision and touch. Neuropsychologia, 39, 1304-1316.

McDonald, J. J., Teder-Sälejärvi, W. A., \& Hillyard, S. A. (2000). Involuntary orienting to sound improves visual perception. Nature, 407, 906-908.

MeltZer, D., \& MasaKi, M. A. (1973). Measures of stimulus control and stimulus dominance. Bulletin of the Psychonomic Society, 1, 28-30.

Morein-Zamir, S., Soto-Faraco, S., \& Kingstone, A. (2003). Auditory capture of vision: Examining temporal ventriloquism. Cognitive Brain Research, 17, 154-163.

ORNE, M. T. (1962). On the social psychology of the psychological experiment: With particular reference to demand characteristics and their implications. American Psychologist, 17, 776-783.

Pallier, C., Dupoux, E., \& Jeannin, X. (1997). EXPE: An expandable programming language for on-line psychological experiments. Behavior Research Methods, Instruments, \& Computers, 29, 322-327.

Partan, S., \& Marler, P. (1999). Communication goes multimodal. Science, 283, 1272-1273.

Pierce, A. H. (1908). The subconscious again. Journal of Philosophy, Psychology, \& Scientific Methodology, 5, 264-271.

Posner, M. I., Nissen, M. J., \& Klein, R. M. (1976). Visual dominance: An information-processing account of its origins and significance. Psychological Review, 83, 157-171.

Posner, M. I., Nissen, M. J., \& Ogden, W. C. (1978). Attended and unattended processing modes: The role of set for spatial location. In H. L. Pick, Jr. \& E. Saltzman (Eds.), Modes of perceiving and processing information (pp. 137-157). Hillside, NJ: Erlbaum.

Quinlan, P. [T.] (2000). The "late" locus of visual dominance. Abstracts of the Psychonomic Society, 5, 64.

Quinlan, P. T., \& Bailey, P. J. (1995). An examination of attentional control in the auditory modality: Further evidence for auditory orienting. Perception \& Psychophysics, 57, 614-628.

Randich, A., Klein, R. M., \& LoLordo, V. M. (1978). Visual dominance in the pigeon. Journal of the Experimental Analysis of Behavior, 30, 129-137.

Rees, G., Frith, C. D., \& Lavie, N. (2001). Processing of irrelevant visual motion during performance of an auditory task. Neuropsychologia, 39, 937-949.

ReEs, G., Russell, C., Frith, C. D., \& Driver, J. (1999). Inattentional blindness versus inattentional amnesia for fixated but ignored words. Science, 286, 2504-2507.

Rock, I., \& Harris, C. S. (1967, May 17). Vision and touch. Scientific American, 216, 96-104.

Rock, I., \& VICTOR, J. (1964). Vision and touch: An experimentally created conflict between the two senses. Science, 143, 594-596.

RodwAY, P. (2005). The modality shift effect and the effectiveness of warning signals in different modalities. Acta Psychologica, 120, 199-226.

Sekuler, R., Sekuler, A. B., \& Lau, R. (1997). Sound alters visual motion perception. Nature, $\mathbf{3 8 5}, 308$.

Shams, L., Kamitani, Y., \& Shimojo, S. (2000). Illusions: What you see is what you hear. Nature, $\mathbf{4 0 8}, 788$.

Shapiro, K. L., Egerman, B., \& Klein, R. M. (1984). Effects of arousal on human visual dominance. Perception \& Psychophysics, $35,547-552$

Shapiro, K. L., Jacobs, W. J., \& LoLordo, V. M. (1980). Stimulusreinforcer interactions in Pavlovian conditioning of pigeons: Implications for selective associations. Animal Learning \& Behavior, 8, 586-594.

SHAPIRO, K. L., \& JoHnSON, T. L. (1987). Effects of arousal on attention to central and peripheral visual stimuli. Acta Psychologica, 66, 157-172.

Sinnett, S., Costa, A., \& Soto-Faraco, S. (2006). Manipulating inattentional blindness within and across sensory modalities. Quarterly Journal of Experimental Psychology, 59, 1425-1442.

SNOdGrass, J. G., \& VANDERWART, M. (1980). A standardized set of 260 pictures: Norms for name agreement, image agreement, familiarity, and visual complexity. Journal of Experimental Psychology: Human Learning \& Memory, 6, 174-215.

Spence, C., Nicholls, M. E. R., \& Driver, J. (2001). The cost of expecting events in the wrong sensory modality. Perception \& Psychophysics, 63, 330-336.

Spence, C., Shore, D. I., \& Klein, R. M. (2001). Multisensory prior entry. Journal of Experimental Psychology: General, 130, 799-832.

Spence, C., \& SQuire, S. (2003). Multisensory integration: Maintaining the perception of synchrony. Current Biology, 13, R519-R521.

TitCHENER, E. B. (1908). Lectures on the elementary psychology of feeling and attention. New York: Macmillan.

Turatto, M., Benso, F., Galfano, G., \& Umiltà, C. (2002). Nonspatial attentional shifts between audition and vision. Journal of Experimental Psychology: Human Perception \& Performance, 28, 628-639.

Uetake, K., \& Kudo, Y. (1994). Visual dominance over hearing in feed acquisition procedure of cattle. Applied Animal Behavior Science, 42, $1-9$.

WELCH, R. B., \& WARREN, D. H. (1980). Immediate perceptual response to intersensory discrepancy. Psychological Bulletin, 88, 638-667.

Welch, R. B., \& WarReN, D. H. (1986). Intersensory interactions. In K. R. Boff, L. Kaufman, \& J. P. Thomas (Eds.), Handbook of perception and human performance: Vol. 1. Sensory processes and perception (chap. 25, pp. 1-36). New York: Wiley.

Whipple, G. M., Sanford, E. C., \& Colegrove, F. W. (1899). On nearly simultaneous clicks and flashes: The time required for recognition: Notes on mental standards of length. American Journal of Psychology, 10, 280-295.

\section{NOTES}

1. In fact, the somewhat unorthodox procedure used by Colavita (1974), together with the less than impressive results reported in experi- 
ments using simple stimuli (see, e.g., Egeth \& Sager, 1977), may help to explain why there has been so little research on this potentially fascinating topic in recent years.

2. Moreover, this spatial separation between the source of the auditory and visual stimuli may inadvertently have tapped into the effects of anxiety on responding to centrally as opposed to peripherally presented stimuli (see, e.g., Eimer, 2000; Kanwisher, 2001; cf. Shapiro \& Johnson, 1987).

3 . We thank an anonymous reviewer for pointing out the possibility that, if Posner et al. (1976) were correct in claiming that attention is biased to the visual modality in order to compensate for poor alerting abilities of vision, adjusting the relative intensity of the auditory stimuli should not have any consequence for the Colavita effect, as the sensitivity to visual stimuli is of more importance.

(Manuscript received October 7, 2005; revision accepted for publication September 5, 2006.) 